\title{
Neutron Background Detection for a Hard X-ray Balloon-borne Polarimeter
}

\author{
Merlin KOLE $^{* a b}$, M. CHAUVIN ${ }^{a b}$, Y. FUKAZAWA ${ }^{c}$, K. FUKUDA $^{d}$ S. ISHIZU ${ }^{d}$, M. \\ JACKSON $^{a b}$, T. KAMAE ${ }^{e}$, N. KAWAGUCHI ${ }^{d}$, T. KAWANO $^{c}$, M. KISS ${ }^{a b}$, V. MIKHALEV ${ }^{a b}$, \\ E. MORETTI ${ }^{a b}$, M. PEARCE ${ }^{a b}$, S. RYDSTRÖM ${ }^{a b}$, H. TAKAHASHI $^{c}$, T. YANAGIDA ${ }^{f}$
}

${ }^{a}$ The Royal Institute of Technology (KTH), Department of Physics 10691 Stockholm, Sweden

${ }^{b}$ The Oskar Klein Centre for Cosmoparticle Physics, AlbaNova University Centre 10691

Stockholm, Sweden

${ }^{c}$ Department of Physical Science, Hiroshima University Hiroshima 739-8526, Japan

${ }^{d}$ Tokuyama Corporation Shunan, Yamaguchi, Japan

${ }^{e}$ University of Tokyo, Deptartment of Physics, 113-0033 Tokyo, Japan

${ }^{f}$ Kyushu Institute of Technology, Kitakyushu, Fukuoka, Japan

\begin{abstract}
PoGOLite is a balloon-borne hard X-ray polarimeter. It determines polarisation by measuring the azimuthal angular distribution of Compton scattered photons in a plastic scintillator array. The use of an all-plastic target yields a relatively large, low-mass detection area. The dominant source of background for these measurements has been shown, through Geant4 simulations, to originate from high energy ( $\mathrm{MeV}$ range) atmospheric neutrons. Neutrons can pass the instrument's Bismuth Germanium Oxide (BGO) anti-coincidence shield undetected and subsequently scatter between plastic scintillator elements to produce a polarisation signature. A passive $15 \mathrm{~cm}$ thick polyethylene shield surrounding the polarimeter reduces the neutron induced background by an order of magnitude. The background level remains however significant, prompting the need for active monitoring of the continuously changing neutron flux. For this purpose PoGOLite makes use of a phoswich scintillator cell. The phoswich cell consists of a $5 \mathrm{~mm}$ thick Lithium Calcium Aluminium Fluoride (LiCAF) scintillator, used for neutron detection. The LiCAF is surrounded by a BGO anti-coincidence system. This small light weight detector can therefore be used to measure the neutron flux even in high radiation environments. This type of neutron detector was tested on a separate dedicated stratospheric balloon mission in March 2013, called PoGOLino, prior to the PoGOLite flight which took place in July 2013. Results from the test flight and implications for the measurements performed on the PoGOLite flight will be discussed.
\end{abstract}

Technology and Instrumentation in Particle Physics 2014,

2-6 June, 2014

Amsterdam, the Netherlands

\footnotetext{
*Speaker.
} 


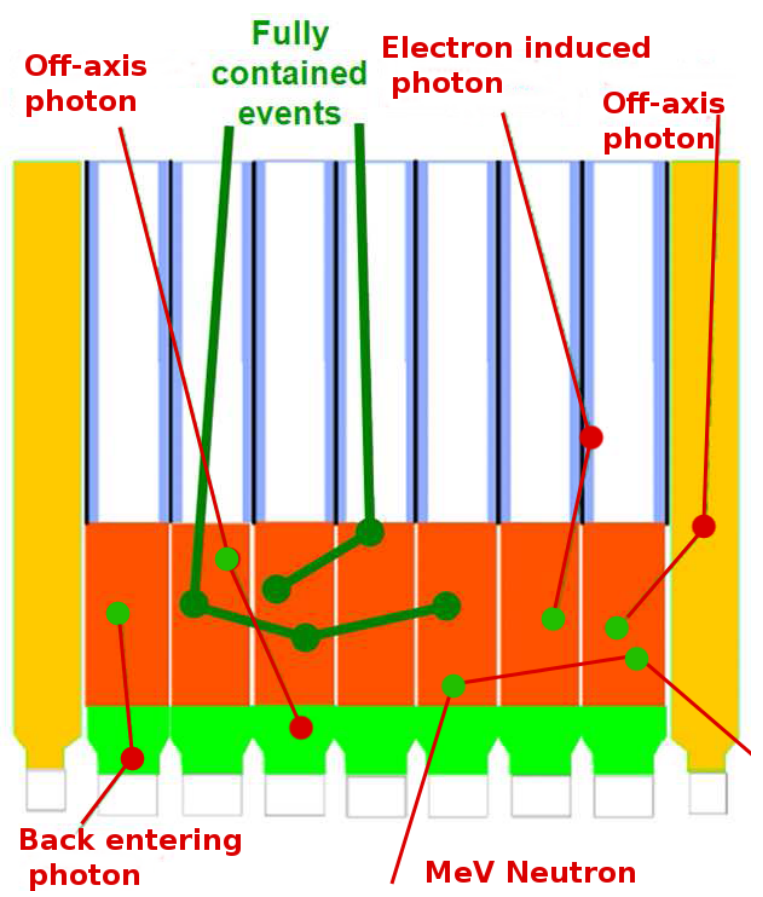

Figure 1: A schematic overview of the PoGOLite instrument showing the various kinds of signal and background events. The plastic scintillator array is shown in orange, the passive and active collimators in black and blue respectively, the bottom and side BGO anti-coincidence systems in green and yellow respectively. Based on a figure as published in [2].

\section{PoGOLite}

PoGOLite is a balloon-borne hard X-ray telescope which aims to measure the polarisation of $\mathrm{X}$-rays in the $20-100 \mathrm{keV}$ energy range [1]. The polarimeter is optimised to observe point sources such as the Crab Nebula and Cygnus-X1. The X-ray polarisation is measured by detecting both the Compton scattering and the subsequent photoabsorption location of X-rays in a segmented scintillator array. The Compton scattering angle, which holds information on the polarisation properties of the X-ray, is measured using the relative positions of the two interaction sites. Plastic was chosen as the detector material to maximise the Compton scattering cross section for X-rays with energies in the $20-100 \mathrm{keV}$ range.

The requirement for both a Compton scattering interaction and a subsequent photoabsorption in different scintillators results in a relatively low signal rate for such polarisation measurements. The low signal rate and the need to perform the measurements in the high radiation environments found at the top of the atmosphere prompt the use of dedicated background rejection systems. For this purpose PoGOLite employs both active and passive collimators which reduce the field of view of the instrument to approximately $2^{\circ}$, thereby optimising the instrument for point sources. Furthermore the plastic scintillator array is enclosed by a Side Anti-coincidence Shield (SAS) and a bottom anti-coincidence shield, both consisting of Bismuth Germanium Oxide (BGO) scintillator crystals. A schematic overview of the PoGOLite instrument can be seen in Figure 1. The combination of these two background rejection systems was shown, through simulations, to minimise the charged 
particle and photon induced background. Neutrons, however, have a decreasing elastic scattering cross section with increasing atomic number, $\mathrm{Z}$, of the material. As a result, the anti-coincidence system of PoGOLite is inefficient for the detection of neutrons entering the instrument, whereas the elastic scattering cross section for neutrons with the plastic scintillator array is significant. For neutrons with energies in the range $0.5-500 \mathrm{MeV}$ the energy deposited in the plastic scintillators by elastic scattering is in the $\mathrm{keV}$ energy range, making such events indistinguishable from valid signal events.

In order to reduce the background induced by neutrons the polarimeter is shielded by approximately $300 \mathrm{~kg}$ of polyethylene. The purpose of the polyethylene shield is to reduce the energy of incoming neutrons to below $\sim 0.5 \mathrm{MeV}$. Geant 4 [3] simulations have shown that the polyethylene shield reduces the background induced by high energy neutrons by an order of magnitude. The remaining background level is, however, still of the same order of magnitude as the signal rate when observing the Crab Nebula. It is therefore essential to monitor this background component in order to be able to account for it during the data analysis phase of the project.

\section{Variations of the Neutron Induced Background}

The fast neutrons responsible for the background in PoGOLite have an atmospheric origin. They are produced in the hadronic component of air showers induced by Galactic cosmic rays. The majority of the neutrons is produced at altitudes between 15 and $20 \mathrm{~km}$. After production, these neutrons travel through the atmosphere and lose energy through elastic scattering with atmospheric nuclei. As a result both the neutron energy spectrum and its absolute flux vary with altitude. The atmospheric neutron flux further depends on the magnetic latitude. The Earth's magnetic dipole field shields the Earth more effectively from charged particles at the equator than at the magnetic poles. As a result the atmospheric neutron flux increases with magnetic latitude. The dependencies of the differential neutron flux on both the altitude and the magnetic latitude was modelled in [4]. The dependencies for the differential neutron flux at $1 \mathrm{MeV}$ on these two parameters as calculated by this model are shown in Figure 2.

A third parameter which influences the atmospheric neutron flux is the solar activity. This is due to the modulation of the incoming charged Galactic cosmic ray flux by the solar wind. An increase in solar activity results in a decrease of the atmospheric neutron flux. Lastly it should be noted that both the solar wind and the Earth's magnetic field affect the same range of the charged Galactic cosmic ray spectrum. These two effects are therefore correlated, resulting in a more pronounced solar activity modulation of the neutron flux at the poles than at the equator.

For PoGOLite all the above mentioned parameters vary throughout the flight. The altitude and the magnetic latitude were measured during its 2013 flight by the Esrange Space Centre. The solar activity during this period is deduced from measurement results from the Thule station of the Bartol Neutron Monitor [5]. Using these parameters the integrated neutron flux in the $0.5-500 \mathrm{MeV}$ energy range impinging on the PoGOLite instrument can be calculated using the model described in [4]. The results of this are shown in of Figure 3. The figure shows the changes in flux resulting from the daily altitude variations of the instrument and the dependency on the magnetic latitude, resulting in a maximum flux when the payload is closest to the magnetic north pole. The neutron 


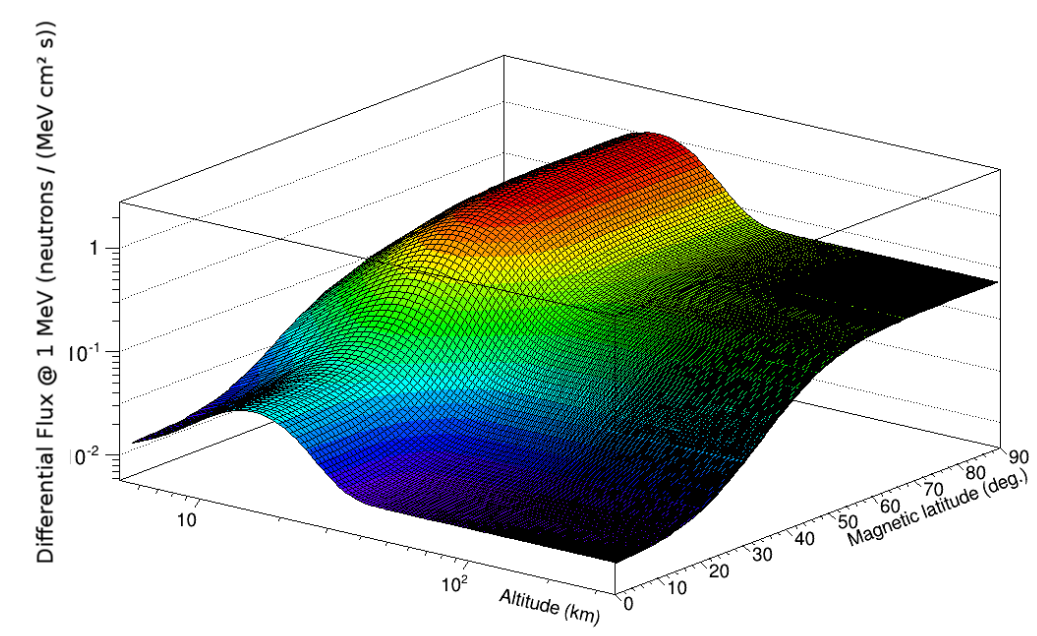

Figure 2: The differential neutron flux at $1 \mathrm{MeV}$ as a function of altitude and latitude as calculated using [4].

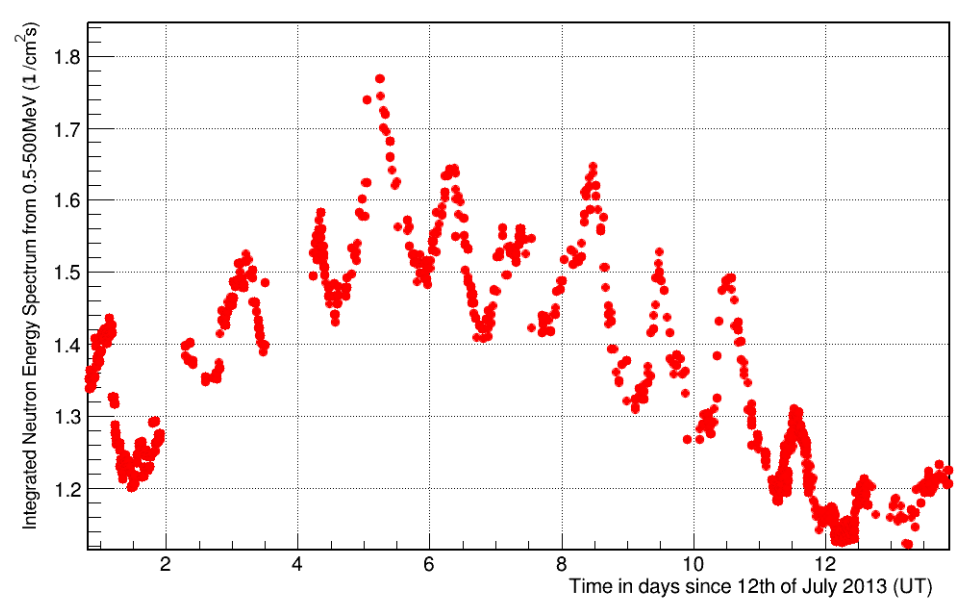

Figure 3: The predicted integrated neutron flux in the energy range of $0.5-500 \mathrm{MeV}$ based on the measured altitude, pressure and the neutron flux as measured on ground, as a function of time (measured in days) for the PoGOLite experiment as calculated using the parametrisation model from [4].

flux can be seen to vary by more than $50 \%$, prompting the need for continuous monitoring of the number of neutrons entering the polarimeter as a function of time.

\section{Neutron Monitor}

PoGOLite monitors the incoming neutron flux using a europium-doped LiCAF crystal with a thickness of $5 \mathrm{~mm}$ provided by the Tokuyama Corporation, Japan. LiCAF is a scintillating material with ${ }^{6} \mathrm{Li}$ embedded in the crystal structure. ${ }^{6} \mathrm{Li}$ has a capture cross section for thermal neutrons of 940 bars. A neutron capture interaction proceeds as: 


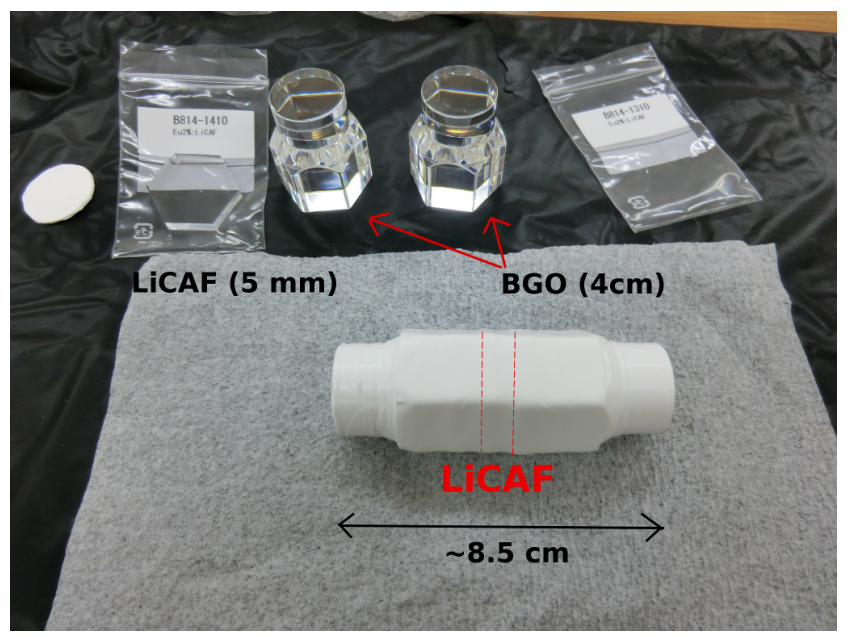

Figure 4: The BGO and LiCAF crystals behind the assembled combination.

$$
\mathrm{n}+{ }^{6} \mathrm{Li} \rightarrow{ }^{4} \mathrm{He}(2.73 \mathrm{MeV})+{ }^{3} \mathrm{H}(2.05 \mathrm{MeV})
$$

The high energy decay products will deposit all their energy within several $\mu \mathrm{m}$ and therefore within the crystal, resulting in a characteristic energy deposition for each neutron capture event. The characteristic energy deposition makes it possible to apply pulse heigh discrimination to distinguish neutron capture events from photon and charged particle interactions in the LiCAF crystal. The efficiency of the pulse height discrimination is further increased by the high light yield of europium-doped LiCAF of $30 \times 10^{4}$ optical photons per neutron capture event [7] with respect to, for example, cerium-doped LiCAF which has a light yield of $3.5 \times 10^{3}$ photons per neutron capture [8]. To further suppress contamination from photon and charged particle interactions in the LiCAF the crystal is sandwiched between two $4 \mathrm{~cm}$ thick BGO crystals, serving as an anti-coincidence shield. The combination of the three crystals is read out by a single Photomultiplier Tube (PMT). Pulse shape discrimination is applied to distinguish between interactions in the BGO and in the LiCAF. The rise times of the two scintillator materials are $300 \mathrm{~ns}$ for BGO and $1600 \mathrm{~ns}$ for LiCAF. The different scintillator crystals and the combination can be seen in Figure 4.

\section{Test Flight}

Prior to the PoGOLite flight the scintillator-based neutron detector was tested on a small balloon-borne experiment called PoGOLino. PoGOLino comprises 2 LiCAF-BGO scintillators combined with a PMT of the type Hamamatsu Photonics R7899EGKNP. The PMTs are read out by an FPGA with a pulse sampling rate of $12.5 \mathrm{MHz}$. Due to the long rise times of the used scintillator materials this relatively low sampling rate is sufficient to perform accurate waveform discrimination on the data [9]. The FPGA is operated through SpaceWire by a PC104 computer connected to ground operations through an Ethernet-over-radio system (E-link) provided by the Esrange Space Centre [6]. One LiCAF-BGO scintillator is embedded in a $8.5 \mathrm{~cm}$ thick polyethylene shield while the second is flown unshielded. The energy dependence of the neutron capture 
cross section of the unshielded detector is similar to that of pure ${ }^{6} \mathrm{Li}$ which is high at sub-eV energies and decreases with increasing energy. The unshielded detector is therefore most efficient for detection of neutrons with energies below $\sim 10 \mathrm{eV}$. The polyethylene shield surrounding the second detector serves to slow down incoming high energy neutrons to energies at which the neutron capture cross section of LiCAF is high. As a result the embedded detector is sensitive up to neutron energies of $1 \mathrm{MeV}$ [9]. The two neutron monitor units, together with an additional scintillator which is not discussed in this paper, the electronics and 8 batteries, type SAFT LSH20, are placed in an aluminium pressure vessel. The pressure vessel makes it possible to operate the electronics at atmospheric pressure. It furthermore provides the possibility to maintain a relatively stable temperature throughout the flight which is important for the measurements due to the large dependency of the scintillation characteristics of BGO on temperature. The temperature stability is further improved by an automated heating unit inside the pressure vessel. The total mass of the instrument is $13 \mathrm{~kg}$. A schematic overview of the instrument can be seen in Figure 5.

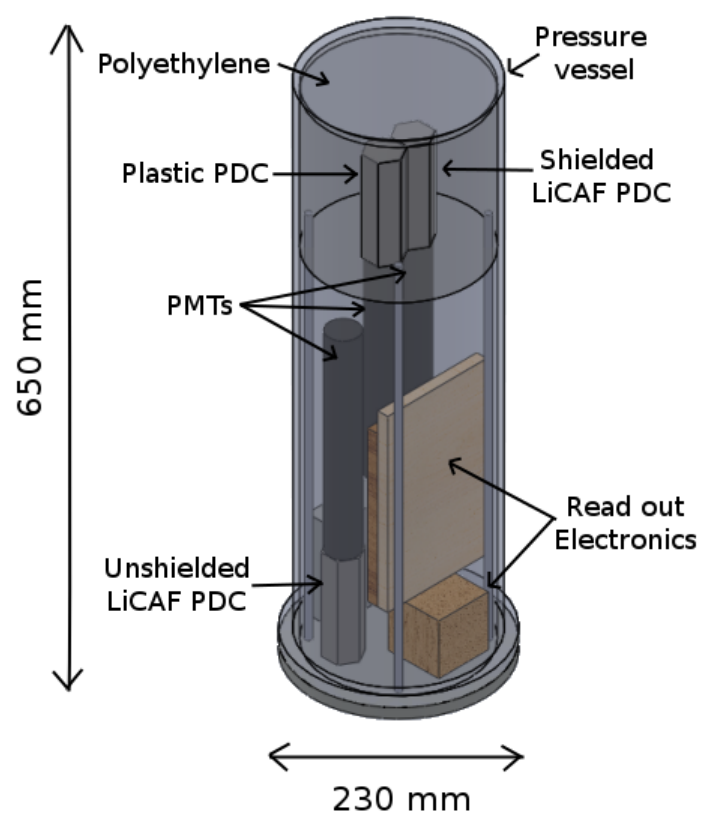

Figure 5: A schematic overview of the PoGOLino instrument containing 3 scintillators, the electronics, the polyethylene shield and the pressure vessel. Taken from [9].

The PoGOLino instrument was launched from the Esrange Space Centre in Northern Sweden on the 20th of March 2013. After an ascent phase of about 1.5 hours a maximum altitude of 31 $\mathrm{km}$ was reached, the payload stayed at this float altitude for approximately 1 hour. The instrument performed 5 minute measurements continuously from before launch up to the moment the payload was disconnected from the balloon. Spectra as taken during ascent and at float can be seen in Figure 6. The spectrum resulting from events, identified as coming from the BGO, is shown in red. The spectrum resulting from events, identified as coming from LiCAF, is shown in blue. The BGO spectra show a broad spectrum containing a peak resulting from $511 \mathrm{keV}$ photons. The LiCAF spectra show the clearly distinguishable neutron absorption peak. Furthermore the background in 
Unshielded
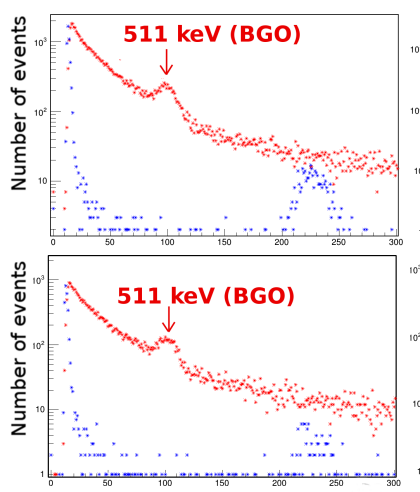

ADC
Shielded

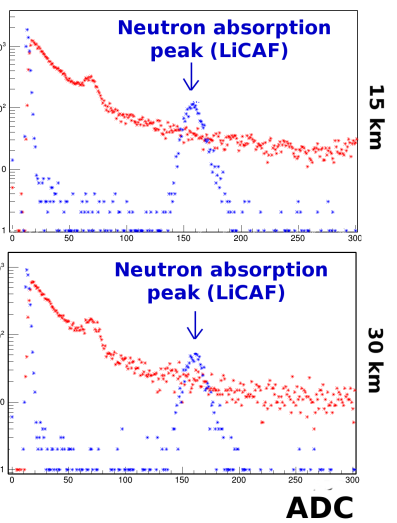

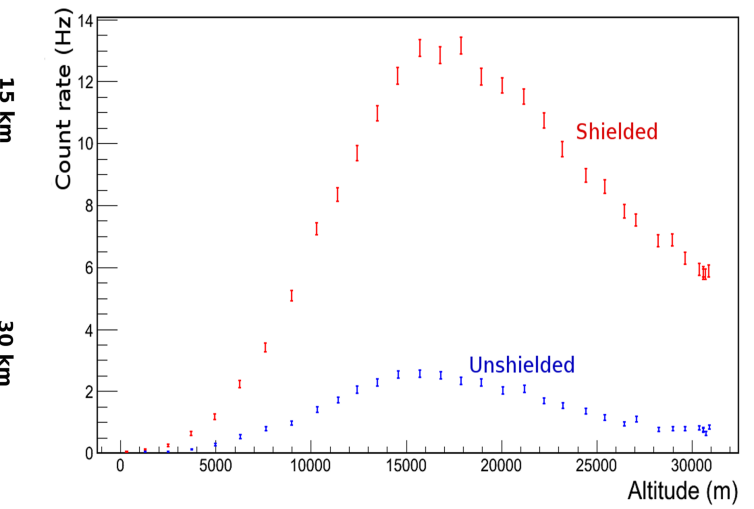

Figure 6: The spectra as measured by the BGO (red) and LiCAF (blue) scintillators on the unshielded (left) and shielded detector (right) on the PoGOLino experiment at an altitude of $15 \mathrm{~km}$ (top) and at float (bottom) are shown on the left. On the right the counting rate as a function of altitude is shown for both detectors, shielded in red and unshielded in blue.

the LiCAF channel can be seen to be negligible close to the neutron absorption peak. The neutron rate measurements can therefore be assumed to be uncontaminated by background.

The measured count rates from both detectors are shown as a function of altitude on the right in Figure 6. The data points are the average rates from 5 minute measurements. The error bars show the statistical errors on the measured count rates. The maximum count rate for both detectors is observed at an altitude of approximately $16 \mathrm{~km}$ corresponding to the mean production altitude for neutrons. Above this level the flux decreases with altitude. The results presented in Figure 6 show that the LiCAF-BGO neutron detector can measure the neutron rate accurately with 5 minute measurements, demonstrating the potential for these detectors to be used to measure the neutron flux entering the PoGOLite instrument and its variability with 5 minute intervals.

\section{Conclusions}

Despite the use of both active and passive background rejection systems, atmospheric neutrons produce an irreducible background in the PoGOLite polarimeter. The count rate induced in the instrument by atmospheric neutrons varies with the altitude and magnetic latitude of the payload and with solar activity. Based on data on the position of the payload taken during its 2013 flight, variations of the impinging flux as large as $50 \%$ can be expected. To measure these variations accurately during the flight, a light-weight scintillator-based detector was developed. The detector, which makes use of LiCAF for neutron detection and BGO for background reduction, was tested on a 3 hour balloon flight in March 2013. The instrument measured the neutron flux as a function of altitude in two different energy ranges. The results of the flight indicate that the detector is capable of measuring the neutron count rate accurately with a negligible contamination from charged particles and photons. The potential to monitor the impinging neutron flux during the PoGOLite mission of this small, light weight neutron detector was hereby demonstrated. 


\section{Acknowledgements}

The authors acknowledge: the SSC Esrange Space Centre for providing us with the position and pressure data from the PoGOLite flight of Summer 2013; the Swedish National Space Board for funding both the PoGOLite and the PoGOLino project; the University of Delaware Department of Physics and Astronomy and the Bartol Research Institute who provided the neutron monitor data from the Thule station.

\section{References}

[1] M. Pearce et al., IEEE Nucl. Sci. Symp. Conf. Rec. (2012) 1885-1892, arXiv:1211.5094

[2] M. Kiss, Doctoral Thesis, KTH - Royal Institute of Technology, Stockholm (2011). http://kth.diva-portal.org

[3] J. Allison et al., IEEE Trans. Nucl. Sci. 53 No. 1 (2006) 270

[4] M. Kole et al., A Model of the Cosmic Ray Induced Atmospheric Neutron Environment. Submitted to Astroparticle Physics (2014).

[5] Bartol Neutron Monitor homepage. http://neutronm. bartol.udel.edu/, accessed in March 2014.

[6] L.-O. Jönsson, ESA SP-671, ESAPAC Proc., Bad Reichenhall, Germany (2009)

[7] T. Yanagida et al. Opt. Mater. ,33, 1243-1247 (2011)

[8] T. Yanagida et al. Opt. Mater. ,32, 311-314 (2009)

[9] M. Kole et al., PoGOLino: a scintillator-based balloon-borne neutron detector, Submitted to NIMA (2014) 